International Journal of Recent advances in Physics (IJRAP) Vol.4, No.1, February 2015

\title{
NEW SCENARIO FOR TRANSITION TO SLOW 3-D TURBULENCE PART I.SLOW 1-D TURBULENCE IN NIKOLAEVSKII SYSTEM.
}

\author{
J. Foukzon \\ Israel Institute of Technology,Haifa, Israel
}

\begin{abstract}
:
Analyticalnon-perturbative study of thethree-dimensional nonlinear stochastic partialdifferential equation with additive thermal noise, analogous to thatproposed by V.N.Nikolaevskii [1]-[5] to describelongitudinal seismic waves, ispresented. Theequation has a threshold of short-waveinstability and symmetry, providing longwavedynamics.New mechanism of quantum chaos generating in nonlineardynamical systemswith infinite number of degrees of freedom is proposed. The hypothesis is said, that physical turbulence could be identifiedwith quantum chaos of considered type. It is shown that the additive thermal noise destabilizes dramatically the ground state of theNikolaevskii system thus causing it to make a direct transition from a spatially uniform to a turbulent state.
\end{abstract}

\section{Keywords:}

3D turbulence, chaos, quantum chaos, additive thermal noise, Nikolaevskii system.

\section{Introduction}

In the present work a non-perturbative analyticalapproach to the studying of problemof quantum chaos in dynamical systems withinfinite number of degrees of freedom isproposed.Statistical descriptions of dynamical chaos and investigations of noise effects on chaoticregimes arestudied.Proposed approach also allows estimate the influence of additive (thermal)fluctuations on the processes of formation ofdeveloped turbulence modes in essentially nonlinearprocesses like electro-convection andother. A principal rolethe influence ofthermalfluctuations on thedynamics of some types of dissipative systems inthe approximate environs of derivation rapid of ashort-wave instability was ascertained. Impotentphysicalresults follows from Theorem 2, is illustrated by example of 3D stochastic model system:

$$
\begin{aligned}
& \frac{\partial u_{\eta}(x, t, \varepsilon)}{\partial t}+\Delta {\left[\varepsilon-(1+\Delta)^{2}\right] u_{\eta}(x, t, \varepsilon) } \\
&+\left[\delta_{1} \frac{\partial u_{\eta}(x, t, \varepsilon)}{\partial x_{1}}+\delta_{2} \frac{\partial u_{\eta}(x, t, \varepsilon)}{\partial x_{2}}+\delta_{3} \frac{\partial u_{\eta}(x, t, \varepsilon)}{\partial x_{3}}\right] u_{\eta}(x, t, \varepsilon)+
\end{aligned}
$$

$+f(x, t)-\sqrt{\eta} w(x, t)=0, x \in \mathbb{R}^{3}(1.1)$

$u_{\eta}(x, 0, \varepsilon)=0, w(x, t)=\frac{\partial^{4} W(x, t)}{\partial x_{1} \partial x_{2} \partial x_{3} \partial t}, \eta \ll 1,0<\delta_{j}, j=1,2,3$,

DOI : 10.14810/ijrap.2015.4102 
which was obtained from thenon-stochastic $3 D$ Nikolaevskiimodel:

$\frac{\partial u(x, t, \varepsilon)}{\partial t}+\Delta\left[\varepsilon-(1+\Delta)^{2}\right] u(x, t, \varepsilon)+\left[\delta_{1} \frac{\partial u(x, t, \varepsilon)}{\partial x_{1}}+\delta_{2} \frac{\partial u(x, t, \varepsilon)}{\partial x_{2}}+\delta_{3} \frac{\partial u(x, t, \varepsilon)}{\partial x_{3}}\right] u(x, t, \varepsilon)+f(x, t)$

which is perturbed by additive "small" white noise $\sqrt{\eta} w(x, t)$. And analytical result also illustrated by exampleof $1 D$ stochasticmodel system

$\frac{\partial u_{\eta}(x, t, \varepsilon)}{\partial t}+\Delta\left[\varepsilon-(1+\Delta)^{2}\right] u_{\eta}(x, t, \varepsilon)+\delta \frac{\partial u_{\eta}(x, t, \varepsilon)}{\partial x_{1}} u_{\eta}(x, t, \varepsilon)+f(x, t)-\sqrt{\eta} w(x, t)=0, x \in \mathbb{R}(1.4)$

$u_{\eta}(x, 0, \varepsilon)=0, w(x, t)=\frac{\partial^{2} W(x, t)}{\partial x \partial t}, \eta \ll 1,0<\delta$,

which was obtained from thenon-stochastic 1DNikolaevskiismodel:

$\frac{\partial u(x, t, \varepsilon)}{\partial t}+\Delta\left[\varepsilon-(1+\Delta)^{2}\right] u(x, t, \varepsilon)+\delta \frac{\partial u(x, t, \varepsilon)}{\partial x_{1}} u(x, t, \varepsilon)+f(x, t)=0, u(x, 0, \varepsilon)=0, x \in \mathbb{R},(1.6)$

$u(x, 0, \varepsilon)=0 .(1.7)$

Systematic study of a different type of chaos at onset "soft-mode turbulence" based onnumerical integration of the simplest 1DNikolaevskii model (1.7)has been executed by many authors [2]-[7].There is an erroneous belief that such numerical integration gives a powerful analysisismeans of the processes of turbulence conception, based on the classical theory ofchaos of the finite-dimensional classical systems [8]-[11].

Remark1.1.However, as it well known, such approximations correct only in a phase ofturbulence conception, when relatively small number of the degrees of freedom excites. In general case, when a very large number of the degrees of freedom excites, well known phenomena of thenumerically induced chaos, can to spoils in the uncontrollable wayany numerical integration[12]-[15]

Remark1.2. Other non trivial problem stays from noise roundoff error in computer computation using floatingpoint arithmetic [16]-[20].In any computer simulation the numerical solution is fraught with truncation by roundoff errors introduced by finite-precision calculation of trajectories of dynamical systems, where roundoff errors or other noise can introduce new behavior and this problem is a very more pronounced in the case of chaotic dynamical systems, because the trajectories of such systems exhibitextensivedependence on initial conditions. As a result, a small random truncation or roundoff error, made computational error at any step of computation will tend to be large magnified by future computationalof the system[17].

Remark1.3.As it well known, if the digitized or rounded quantity is allowed to occupy the nearest of a large number of levels whose smallest separation is $E_{0}$, then, provided that the

original quantity is large compared to $E_{0}$ and is reasonably well behaved, theeffect of the quantization or rounding may betreated as additive random noise [18].Bennett has shown that such additive noise is nearly white, withmean squared value of $E_{0}^{2} / 12[19]$. However the complete uniform white-noise model to be valid in the sense of weak convergence of probabilistic measures as the lattice step tends to zero if the matrices of realization of the system in the state space satisfy certain nonresonance conditions and the finite-dimensional distributions of the input signal are absolutely continuous[19].

The method deprived of these essential lacks in general case has been offered by the author in papers 
[23]-[27].

Remark1.4.Thus from consideration above it is clear thatnumerical integration procedure ofthe 1D Nikolaevskii model (1.6)-(1.7) executed in papers [2]-[7]in fact dealing withstochastic model (1.4)-(1.5).There is an erroneous the point of view,that a white noise with enough small intensity does not bringanysignificant contributions in turbulent modes, see for example [3]. By this wrong assumptions the results of the numerical integration procedure ofthe1D Nikolaevskii model (1.6)-(1.7) were mistakenly considered and interpretedas a veryexact modeling the slow turbulence within purely non stochastic Nikolaevskii model (1.6)-(1.7).Accordingly wrongconclusionsabout that temperature noisesdoes not influence slowturbulence have been proposed in [3]. However in [27] has shown non-perturbativelythat that a white noise with enough small intensity can to bring significant contributions in turbulent modes and even to change this modes dramatically.

At the present time it is generally recognized thatturbulence in its developed phase has essentiallysingular spatially-temporal structure. Such asingular conduct is impossible to describeadequately by the means of some model system of equations of a finite dimensionality. In thispoint a classical theory of chaos is able todescribe only small part of turbulencephenomenon in liquid and another analogous sof dynamical systems. The results of non-perturbative modeling ofsuper-chaotic modes, obtained in the present paper allow us to put out a quite probablehypothesis: developed turbulence in the realphysical systems with infinite number of degreesof freedom is a quantum super-chaos, at that the quantitative characteristics of this super-chaos, iscompletely determined by non-perturbativecontribution of additive (thermal) fluctuations inthe corresponding classical system dynamics [18]-[20].

\section{Main Theoretical Results}

We study the stochastic $r$-dimensionaldifferential equation analogous proposed byNikolaevskii [1] to describe longitudinal seismicwaves:

$$
\begin{aligned}
& \frac{\partial u_{\eta}(x, t, \varepsilon, \omega)}{\partial t}+\Delta\left[\varepsilon-(1+\Delta)^{2}\right] u_{\eta}(x, t, \varepsilon, \omega)+u_{\eta}(x, t, \varepsilon, \omega) \sum_{j=1}^{r} \delta_{i} \frac{\partial u_{\eta}(x, t, \varepsilon, \omega)}{\partial x_{i}}+f(x, t)- \\
& \sqrt{\eta} w(x, t, \omega)=0,(2.1) \\
& x \in \mathbb{R}^{r}, u_{\eta}(x, 0, \varepsilon, \omega)=0, w(x, t)=\frac{\partial^{r+1} W(x, t)}{\partial x_{1} \partial x_{2} \ldots \partial x_{r} \partial t}, 0<\delta_{j}, j=1, \ldots, r .(2.2)
\end{aligned}
$$

The main difficulty with the stochasticNikolaevskii equationis that the solutions do not take values in an function space but in generalized functionspace. Thus it is necessary to give meaning to the non-linear terms $\partial_{x_{j}} u_{\eta}^{2}(x, t, \varepsilon, \omega), j=1, \ldots, r$ because the usual product makes no sense for arbitrary distributions. We deal with product of distributions via regularizations, i.e., we approximate the distributions by appropriate way and pass to the limit.In this paper we use the approximation of the distributions by approach of Colombeaugeneralized functions [28].

Notation 2.1. We denote by $\mathcal{D}\left(\mathbb{R}^{r} \times \mathbb{R}_{+}\right)$the space of the infinitely differentiable functionswith compact supportin $\mathbb{R}^{r} \times \mathbb{R}_{+}$andby $\mathcal{D}^{\prime}\left(\mathbb{R}^{r} \times \mathbb{R}_{+}\right)$its dual space.Let $\mathfrak{C}=(\Omega, \Sigma, \mu)$ be a probability space. We denote by $\mathbf{D}$ the space of allfunctions $T: \Omega \rightarrow \mathcal{D}^{\prime}\left(\mathbb{R}^{r} \times \mathbb{R}_{+}\right)$such that $\langle T, \varphi\rangle$ is a random variable for all $\varphi \in$ $\mathcal{D}\left(\mathbb{R}^{r} \times \mathbb{R}_{+}\right)$.Theelements ofDare called random generalized functions.

Definition 2.1.[29].We say that a random field $\left\{\Re(x, t) \mid t \in \mathbb{R}_{+}, x \in \mathbb{R}^{r}\right\}$ isa spatiallydependent semimartingale if for each $x \in \mathbb{R}^{r},\left\{\Re(x, t) \mid t \in \mathbb{R}_{+}\right\}$is asemimartingale in relation to the same filtration $\left\{\mathcal{F}_{t} \mid t \in \mathbb{R}_{+}\right\}$. If $\Re(x, t)$ is a $C^{\infty}$-function of $x$ and continuous inalmost everywhere, it is called $\mathrm{a} C^{\infty}$-semimartingale. 
Definition 2.2.We say that that $u_{\eta}(x, t, \varepsilon, \omega) \in \mathbf{D}$ is a strong generalized solution(SGS) of the Eq.(2.1)-(2.2) if there exists asequence of $C^{\infty}$-semimartingales $u_{\eta}(x, t, \varepsilon, \epsilon, \omega), \epsilon \in(0,1]$ such that there exists

(i) $u_{\eta}(x, t, \varepsilon, \omega)=_{\text {def }} \lim _{\epsilon \rightarrow 0} u_{\eta}(x, t, \varepsilon, \epsilon, \omega) \operatorname{in} \mathcal{D}^{\prime}\left(\mathbb{R}^{r} \times \mathbb{R}_{+}\right)$almost surely for $\omega \in \Omega$,

(ii) $\partial_{x_{j}} u_{\eta}^{2}(x, t, \varepsilon, \epsilon, \omega)={ }_{\operatorname{def}} \lim _{\epsilon \rightarrow 0} \partial_{x_{j}} u_{\eta}^{2}(x, t, \varepsilon, \epsilon, \omega), j=1, \ldots$, ralmost surely for $\omega \in \Omega$,

(iii)for all $\varphi \in \mathcal{D}\left(\mathbb{R}^{r} \times \mathbb{R}_{+}\right)$

$$
\begin{aligned}
\text { (iv) }\left\langle\partial_{t} u_{\eta}(x, t, \varepsilon, \omega), \varphi\right\rangle-\left\langle\Delta\left[\varepsilon-(1+\Delta)^{2}\right] u_{\eta}(x, t, \varepsilon, \omega), \varphi\right\rangle-\sum_{j=1}^{r} \frac{\delta_{i}}{2}\left\langle\partial_{x_{j}} u_{\eta}^{2}(x, t, \varepsilon, \omega), \varphi\right\rangle \\
-\langle f(x, t), \varphi\rangle+
\end{aligned}
$$

$+\sqrt{\eta} \int_{\mathbb{R}^{r}} d x \int_{0}^{\infty} \varphi(t, x) d W_{t}(x, t)=0, t \in \mathbb{R}_{+}$almost surely for $\omega \in \Omega$,

and where $W_{t}(x, t)=\frac{\partial^{r} W(x, t)}{\partial x_{1} \partial x_{2} \ldots \partial x_{r}}$

(v) $u_{\eta}(x, t, \varepsilon, \omega)=0$ almost surely for $\omega \in \Omega$.

However in this paper we use the solutionsofstochastic Nikolaevskii equation only in the sense of Colombeaugeneralized functions [30].

Remark2.1.Note that from Definition 2.2it is clear that any strong generalized solution $u_{\eta}(x, t, \varepsilon, \omega)$ of the Eq.(2.1)-(2.2) one can to recognized as Colombeaugeneralized function such that

$$
u_{\eta}(x, t, \varepsilon, \omega)=_{\operatorname{def}}\left(u_{\eta}(x, t, \varepsilon, \epsilon, \omega)\right)_{\epsilon}(\#)
$$

By formula (\#)one can todefine appropriate generalized solutionof the Eq.(2.1)-(2.2) even if a strong generalized solutionof the Eq.(2.1)-(2.2) does not exist.

Definition 2.3.Assumethata strong generalized solution of the Eq.(2.1)-(2.2) does not exist.We shall say that:

(I)Colombeaugeneralized stochastic process $\left(u_{\eta}(x, t, \varepsilon, \epsilon, \omega)\right)_{\epsilon}$ is a weak generalized solution (WGS) of the Eq.(2.1)-(2.2) orColombeausolutionof the Eq.(2.1)-(2.2) iffor all $\varphi \in \mathcal{D}\left(\mathbb{R}^{r} \times \mathbb{R}_{+}\right)$andfor all $\epsilon \in(0,1]$

$$
\begin{gathered}
\text { (i) }\left\langle u_{\eta}(x, t, \varepsilon, \epsilon, \omega), \partial_{t} \varphi\right\rangle-\left\langle\Delta\left[\varepsilon-(1+\Delta)^{2}\right] u_{\eta}(x, t, \varepsilon, \epsilon, \omega), \varphi\right\rangle-\sum_{j=1}^{r} \frac{\delta_{i}}{2}\left\langle\partial_{x_{j}} u_{\eta}^{2}(x, t, \varepsilon, \epsilon, \omega), \varphi\right\rangle+ \\
+\langle f(x, t), \varphi\rangle+\sqrt{\eta} \int_{\mathbb{R}^{r}} d x \int_{0}^{\infty} \varphi(t, x) d W_{t}(x, t)=0, t \in \mathbb{R}_{+} \text {almost surely for } \omega \in \Omega,
\end{gathered}
$$

(ii) $u_{\eta}(x, t, \varepsilon, \epsilon, \omega)=0$ almost surely for $\omega \in \Omega$. 
(II)Colombeaugeneralized stochastic process $\left(u_{\eta}(x, t, \varepsilon, \epsilon, \omega)\right)_{\epsilon}$ is aColombeau-Ito'ssolutionof the Eq.(2.1)-(2.2) if for all $\varphi \in \mathcal{D}\left(\mathbb{R}^{r}\right)$ and for all $\epsilon \in(0,1]$

$$
\text { (i) }\left\langle\partial_{t} u_{\eta}(x, t, \varepsilon, \epsilon, \omega), \varphi\right\rangle+\left\langle\Delta\left[\varepsilon-(1+\Delta)^{2}\right] u_{\eta}(x, t, \varepsilon, \epsilon, \omega), \varphi\right\rangle \sum_{j=1}^{r} \frac{\delta_{i}}{2}\left\langle\partial_{x_{j}} u_{\eta}^{2}(x, t, \varepsilon, \epsilon, \omega), \varphi\right\rangle-
$$

$-\langle f(x, t), \varphi\rangle-\sqrt{\eta} \int_{\mathbb{R}^{r}} \varphi(x) w(x, t) d x=0, t \in \mathbb{R}_{+}$almost surely for $\omega \in \Omega$,

(ii) $u_{\eta}(x, t, \varepsilon, \epsilon, \omega)=0$ almost surely for $\omega \in \Omega$.

Notation 2.2. [30]. Thealgebra of moderate element we denote by $\mathcal{E}_{M}\left[\mathbb{R}^{r}\right]$.The Colombeau algebra of the Colombeau generalized functionwe denote by $\mathcal{G}\left(\mathbb{R}^{r}\right)$.

Notation 2.3.[30].We shall use the following designations. If $U \in \mathcal{G}\left(\mathbb{R}^{r}\right)$ it representatives will be denoted by $R_{U}$, their values on $\varphi=\left(\varphi_{\epsilon}(x)\right), \epsilon \in(0,1]$ will be denoted by $R_{U}(\varphi)$ and it pointvalues at $x \in \mathbb{R}^{r}$ will be denoted $R_{U}(\varphi, x)$.

Definition 2.4.[30].Let $A_{0}=A_{0}\left(\mathbb{R}^{r}\right)$ be the set of all $\varphi \in D\left(\mathbb{R}^{r}\right)$ such that $\int \varphi(x) d x=1$. Let $\mathbb{C}=(\Omega, \Sigma, \mu)$ be a probability space.Colombeau random generalized function this is a map $U: \Omega \rightarrow \mathcal{G}\left(\mathbb{R}^{r}\right)$ such that there is representing function $R_{U}: A_{0} \times \mathbb{R}^{r} \times \Omega$ with the properties:

(i) for fixed $\varphi \in A_{0}\left(\mathbb{R}^{r}\right)$ the function $(x, \omega) \rightarrow R_{U}(\varphi, x, \omega)$ is a jointly measurable on $\mathbb{R}^{r} \times \Omega$;

(ii) almost surely in $\omega \in \Omega$,the function $\varphi \rightarrow R_{U}(\varphi, ., \omega)$ belongs to $\mathcal{E}_{M}\left[\mathbb{R}^{r}\right]$ and is a representative of $U$;

Notation 2.3.[30]. TheColombeaualgebra ofColombeau random generalized functionis denoted $\operatorname{by} \mathcal{G}_{\Omega}\left(\mathbb{R}^{r}\right)$.

Definition 2.5.Let $\mathfrak{C}=(\Omega, \Sigma, \mu)$ be a probability space. Classically, a generalizedstochastic process on $\mathbb{R}^{r}$ is a weakly measurable map $V: \Omega \rightarrow D^{\prime}\left(\mathbb{R}^{r}\right)$ denoted by $V \in D_{\Omega}^{\prime}\left(\mathbb{R}^{r}\right)$. If $\varphi \in A_{0}\left(\mathbb{R}^{r}\right)$, then

(iii) $\quad V(\omega) * \varphi(x)=\langle V(\omega), \varphi(x-)$.$\rangle is a measurable with respect to \omega \in \Omega$ and

(iv) smooth with respect to $x \in \mathbb{R}^{r}$ and hence jointly measurable.

(v) Also $(V(\omega) * \varphi(x)) \in \mathcal{E}_{M}\left[\mathbb{R}^{r}\right]$.

(vi) Therefore $R_{V}(\varphi, x, \omega)=V(\omega) * \varphi(x)$ qualifies as an representing function for an element of $\mathcal{G}_{\Omega}\left(\mathbb{R}^{r}\right)$.

(vii) In this way we have an imbedding $\mathcal{D}^{\prime}\left(\mathbb{R}^{r}\right) \rightarrow \mathcal{G}_{\Omega}\left(\mathbb{R}^{r}\right)$.

Definition 2.6. Denote by $S(T)=S\left(\mathbb{R}^{r+1}\right) \uparrow T$ the space of rapidly decreasing smooth functions on $T=\mathbb{R}^{r} \times[0, \infty)$. Let $\mathbb{C}=(\Omega, \Sigma, \mu)$ with (i) $\Omega=S^{\prime}(T)$, (ii) $\Sigma$ - the Borel $\sigma$-algebra generated by the weak topology. Therefore there is unique probability measure $\mu$ on $(\Omega, \Sigma)$ such that

$$
\int d \mu(\omega) \exp [i\langle\omega, \varphi\rangle]=\exp \left(-\frac{1}{2}\|\varphi\|_{L_{2}(T)}^{2}\right)
$$

for all $\varphi \in S(T)$. White noise $w(\omega)$ with the support in $T$ is the generalized process $w(\omega): \Omega \rightarrow$ $\mathcal{D}^{\prime}\left(\mathbb{R}^{r+1}\right)$ such that: (i) $w(\varphi)=\langle w(\omega), \varphi\rangle=\langle\omega, \varphi \uparrow T\rangle \quad$ (ii) $\mathbf{E}[w(\varphi)]=0, \quad$ (iii) $\quad \mathbf{E}\left[w^{2}(\varphi)\right]=$ $\|\varphi\|_{L_{2}(T)}^{2}$.Viewed as a Colombeau random generalized function, it has a representative (denoting on variables in $\mathbb{R}^{r+1}$ by $\left.(x, t)\right): R_{w}(\varphi, x, t, \omega)=\langle\omega, \varphi(x-, t-) \uparrow T\rangle$, which vanishes if $t$ is less than minus the diameter of the support of $\varphi$. Thereforew is a zero on $\mathbb{R}^{r} \times(-\infty, 0)$ in $\mathcal{G}_{\Omega}\left(\mathbb{R}^{r+1}\right)$. Note that its variance is the Colombeau constant: $\mathbf{E}\left[R_{w}^{2}(\varphi, x, t, \omega)\right]=\int_{\mathbb{R}^{r}} d y \int_{0}^{\infty}|\varphi(x-y, t-s)|^{2} d s$. 
Definition 2.7.Smoothedwith respect to $\mathbb{R}^{r}$ white noise $\left(w_{\epsilon}(x, t)\right)_{\epsilon}$ the representative $R_{w}(\varphi, x, t, \omega)$ with $\varphi \in A_{0}\left(\mathbb{R}^{r}\right) \times \mathcal{D}^{\prime}\left(\mathbb{R}^{r}\right)$, such that $\varphi=\left(\varphi_{\epsilon}(x)\right), \epsilon \in(0,1]$ and $\varphi_{\epsilon}=$ $\epsilon^{-r} \varphi\left(\frac{x}{\epsilon}\right) \delta(t)$.

Theorem 2.1.[25]. (Strong Large Deviation Principle fo SPDE)(I) $\operatorname{Let}\left(u_{\epsilon}(x, t, \varepsilon, \eta, \omega)\right)_{\epsilon}, \epsilon \in$ $(0,1]$ be solution of theColombeau-Ito'sSPDE [26]:

$$
\begin{aligned}
& \frac{\partial\left(u_{\epsilon}(x, t, \varepsilon, \eta, \omega)\right)_{\epsilon}}{\partial t}+\Delta\left[\varepsilon-(1+\Delta)^{2}\right]\left(u_{\epsilon}(x, t, \varepsilon, \eta, \omega)\right)_{\epsilon}+ \\
& \left(\left(F_{\epsilon}\left(u_{\epsilon}(x, t, \varepsilon, \eta, \omega)\right)\right)_{\epsilon}\right) \sum_{i=1}^{r} \delta_{i}\left(F_{\epsilon}\left(\frac{\partial u_{\epsilon}(x, t, \varepsilon, \eta, \omega)}{\partial x_{i}}\right)\right)_{\epsilon}+\left(f_{\epsilon}(x, t)\right)_{\epsilon}-\sqrt{\eta}\left(w_{\epsilon}(x, t, \omega)\right)_{\epsilon}=0,(2.3) \\
& x \in \mathbb{R}^{r}, u_{\epsilon}(x, t, \varepsilon, \eta, \omega) \equiv 0, \delta_{j}>0, j=1, \ldots, r .(2.4)
\end{aligned}
$$

Here: $(1)\left(F_{\epsilon}(z)\right)_{\epsilon} \in G(\mathbb{R}), G(\mathbb{R})$ the Colombeau algebra of Colombeau generalized functions and $F_{0}(z)=$ $z$.

(2) $\left(f_{\epsilon}(x, t)\right)_{\epsilon} \in \mathcal{G}\left(\mathbb{R}^{r+1}\right)$.

(3) $w_{\epsilon}(x, t)$ is a smoothedwith respect to $\mathbb{R}^{r}$ white noise.

(II)Let $\left(u_{\epsilon, n}\left(x_{n}, t, \varepsilon, \eta, \omega\right)\right)_{\epsilon}, \epsilon \in(0,1]$ be solution of the Colombeau-Ito's SDE[26]:

$\frac{d\left(u_{\epsilon, \boldsymbol{n}}\left(x_{\boldsymbol{n}}, t, \varepsilon, \eta, \omega\right)\right)_{\epsilon}}{d t}+\Delta_{\boldsymbol{n}}\left[\varepsilon-\left(1+\Delta_{\boldsymbol{n}}\right)^{2}\right]\left(u_{\epsilon, \boldsymbol{n}}\left(x_{\boldsymbol{n}}, t, \varepsilon, \eta, \omega\right)\right)_{\epsilon}+$

$\left(\left(F_{\epsilon}\left(u_{\epsilon, \boldsymbol{n}}\left(x_{\boldsymbol{n}}, t, \varepsilon, \eta, \omega\right)\right)\right)_{\epsilon}\right) \sum_{i=1}^{r} \delta_{i}\left(F_{\epsilon}\left(\frac{u_{\epsilon, \boldsymbol{n}+1, i}\left(x_{\boldsymbol{n}+1, i}, t, \varepsilon, \eta, \omega\right)-u_{\epsilon, \boldsymbol{n}}\left(x_{n, i}, t, \varepsilon, \eta, \omega\right)}{h_{N}}\right)\right)_{\epsilon}+\left(f_{\epsilon}\left(x_{\boldsymbol{n}}, t\right)\right)_{\epsilon}-$

$\sqrt{\eta}\left(w_{\epsilon}\left(x_{n}, t, \omega\right)\right)_{\epsilon}=0,(2.5)$

$x_{\boldsymbol{n}} \in \boldsymbol{\Theta} \subset h_{N} \cdot \mathbb{Z}^{r}, \boldsymbol{n}=\left(n_{1}, \ldots, n_{r}\right) \in \mathbb{Z}^{r},|\boldsymbol{n}|=\sum_{i=1}^{r} n_{j}, x_{\boldsymbol{n}+1, i}=\left(x_{n_{1}}, \ldots, x_{n_{i}}+h_{N}, \ldots, x_{n_{r}}\right),-N \leq n_{i} \leq$ $N,(2.6)$

$u_{\epsilon, \boldsymbol{n}}\left(x_{\boldsymbol{n}}, 0, \varepsilon, \eta, \omega\right) \equiv 0, x_{\boldsymbol{n}} \in \boldsymbol{\Theta} .(2.7)$

Here Eq.(2.5)-Eq.(2.7) is obtained from Eq.(2.3)-Eq.(2.4) byspatialdiscretizationon finite lattice $\Theta$ $h_{N} \rightarrow 0$ if $N \rightarrow \infty$ and $\Delta_{n}$-is a latticed Laplacian[31]-[33].

(III)Assume that Colombeau-Ito's SDE (2.5)-(2.7)is a strongly dissipative.[26].

(IV) Let $\Re(x, t, \varepsilon, \lambda)$ be the solutions of thelinear PDE:

$\frac{\partial \Re(x, t, \varepsilon, \lambda)}{\partial t}+\Delta\left[\varepsilon-(1+\Delta)^{2}\right] \Re(x, t, \varepsilon, \lambda)+\lambda \sum_{i=1}^{r} \delta_{i} \frac{\partial \Re(x, t, \varepsilon, \lambda)}{\partial x_{i}}-f(x, t)=0, \lambda \in \mathbb{R},(2.8)$

$\Re(x, 0, \varepsilon, \lambda)=0 .(2.9)$

Then

$\liminf _{\epsilon \rightarrow 0} \mathbf{E}\left[\left|u_{\epsilon}(x, t, \varepsilon, \eta, \omega)-\lambda\right|^{2}\right] \leq \Re(x, t, \varepsilon, \lambda)$. 
Proof.The proof based on Strong large deviations principles(SLDP-Theorem) for Colombeau-Ito'ssolution of the Colombeau-Ito's SDE, see [26],theorem 6.BySLDP-Theorem one obtain directlythe differential master equation (see [26],Eq.(90)) for Colombeau-Ito's SDE(2.5)-(2.7):

$$
\begin{array}{r}
\frac{d\left(U_{\epsilon, \boldsymbol{n}}\left(x_{\boldsymbol{n}}, t, \varepsilon\right)\right)_{\epsilon}}{d t}+\Delta_{\boldsymbol{n}}\left[\varepsilon-\left(1+\Delta_{\boldsymbol{n}}\right)^{2}\right]\left(U_{\epsilon, \boldsymbol{n}}\left(x_{n}, t, \varepsilon\right)\right)_{\epsilon}+ \\
+\lambda_{\boldsymbol{n}} \sum_{i=1}^{r} \delta_{i}\left(F_{\epsilon}\left(\frac{U_{\epsilon, \boldsymbol{n}+1, i}\left(x_{n+1, i} t, \varepsilon\right)-U_{\epsilon, \boldsymbol{n}}\left(x_{n, i}, t, \varepsilon\right)+}{h_{N}}\right)\right)_{\epsilon}+\left(f_{\epsilon}\left(x_{\boldsymbol{n}}, t\right)\right)_{\epsilon}+o(\epsilon)=0,(2.11) \\
U_{\epsilon, \boldsymbol{n}}\left(x_{\boldsymbol{n}}, 0, \varepsilon\right)=-\lambda_{\boldsymbol{n}} .
\end{array}
$$

We set now $\lambda_{n} \equiv \lambda \in \mathbb{R}$. Then from Eq.(2.13)-Eq.(2.14) we obtain

$$
\begin{array}{r}
\frac{d\left(U_{\epsilon, \boldsymbol{n}}\left(x_{\boldsymbol{n}}, t, \varepsilon, \lambda\right)\right)_{\epsilon}}{d t}+\Delta_{\boldsymbol{n}}\left[\varepsilon-\left(1+\Delta_{\boldsymbol{n}}\right)^{2}\right]\left(U_{\epsilon, \boldsymbol{n}}\left(x_{\boldsymbol{n}}, t, \varepsilon, \lambda\right)\right)_{\epsilon}+ \\
+\lambda \sum_{i=1}^{r} \delta_{i}\left(F_{\epsilon}\left(\frac{U_{\epsilon, \boldsymbol{n}+1, i}\left(x_{\boldsymbol{n}+1, i}, t, \varepsilon, \lambda\right)-U_{\epsilon, \boldsymbol{n}}\left(x_{n, i}, t, \varepsilon, \lambda\right)}{h_{N}}\right)\right)_{\epsilon}+\left(f_{\epsilon}\left(x_{\boldsymbol{n}}, t\right)\right)_{\epsilon}+O(\epsilon)=0,(2.13) \\
U_{\epsilon, \boldsymbol{n}}\left(x_{\boldsymbol{n}}, 0, \varepsilon, \lambda\right)=-\lambda .
\end{array}
$$

From Eq.(2.5)-Eq.(2.7) and Eq.(2.13)-Eq.(2.14) by SLDP-Theorem (see see[26], inequality(89)) we obtain the inequality

$$
\liminf _{\epsilon \rightarrow 0} \mathbf{E}\left[\left|u_{\epsilon, n}\left(x_{n}, t, \varepsilon, \eta, \omega\right)-\lambda\right|^{2}\right] \leq U_{\epsilon, \boldsymbol{n}}\left(x_{\boldsymbol{n}}, t, \varepsilon, \lambda\right), \epsilon \in(0,1] .(2.15)
$$

Let us consider now the identity

$$
\left|u_{\epsilon}(x, t, \varepsilon, \eta, \omega)-\lambda\right|^{2}=\left|\left[u_{\epsilon}(x, t, \varepsilon, \eta, \omega)-u_{\epsilon, \boldsymbol{n}}\left(x_{\boldsymbol{n}}, t, \varepsilon, \eta, \omega\right)\right]+\left[u_{\epsilon, \boldsymbol{n}}\left(x_{\boldsymbol{n}}, t, \varepsilon, \eta, \omega\right)-\lambda\right]\right|^{2} .
$$

Fromtheidentity (2.16) bythetriangle inequality we obtaintheinequality

$$
\left|u_{\epsilon}(x, t, \varepsilon, \eta, \omega)-\lambda\right|^{2} \leq\left|u_{\epsilon}(x, t, \varepsilon, \eta, \omega)-u_{\epsilon, \boldsymbol{n}}\left(x_{\boldsymbol{n}}, t, \varepsilon, \eta, \omega\right)\right|^{2}+\left|u_{\epsilon, \boldsymbol{n}}\left(x_{\boldsymbol{n}}, t, \varepsilon, \eta, \omega\right)-\lambda\right|^{2} .
$$

From theidentity (2.17) by integration we obtain theinequality

$$
\begin{gathered}
\mathbf{E}\left|u_{\epsilon}(x, t, \varepsilon, \eta, \omega)-\lambda\right|^{2} \leq \\
\leq \mathbf{E}\left|u_{\epsilon}(x, t, \varepsilon, \eta, \omega)-u_{\epsilon, \boldsymbol{n}}\left(x_{\boldsymbol{n}}, t, \varepsilon, \eta, \omega\right)\right|^{2}+\left|u_{\epsilon, \boldsymbol{n}}\left(x_{\boldsymbol{n}}, t, \varepsilon, \eta, \omega\right)-\lambda\right|^{2} .
\end{gathered}
$$

From theidentity (2.18) by theidentity (2.15) for all $\epsilon \in(0,1]$ we obtain theinequality

$$
\begin{array}{r}
\mathbf{E}\left|u_{\epsilon}(x, t, \varepsilon, \eta, \omega)-\lambda\right|^{2} \leq \\
\leq \mathbf{E}\left|u_{\epsilon}(x, t, \varepsilon, \eta, \omega)-u_{\epsilon, \boldsymbol{n}}\left(x_{\boldsymbol{n}}, t, \varepsilon, \eta, \omega\right)\right|^{2}+U_{\epsilon, \boldsymbol{n}}\left(x_{\boldsymbol{n}}, t, \varepsilon, \lambda\right) .(2.19)
\end{array}
$$


In the limit $N \rightarrow \infty$ fromthe inequality we obtain the inequality

$$
\begin{gathered}
\mathbf{E}\left|u_{\epsilon}(x, t, \varepsilon, \eta, \omega)-\lambda\right|^{2} \leq \\
\leq \limsup _{N \rightarrow \infty} \mathbf{E}\left|u_{\epsilon}(x, t, \varepsilon, \eta, \omega)-u_{\epsilon, \boldsymbol{n}}\left(x_{n}, t, \varepsilon, \eta, \omega\right)\right|^{2}+\limsup _{N \rightarrow \infty} U_{\epsilon, \boldsymbol{n}}\left(x_{\boldsymbol{n}}, t, \varepsilon, \lambda\right) .
\end{gathered}
$$

Wenote that

$\limsup _{N \rightarrow \infty} \mathbf{E}\left|u_{\epsilon}(x, t, \varepsilon, \eta, \omega)-u_{\epsilon, \boldsymbol{n}}\left(x_{\boldsymbol{n}}, t, \varepsilon, \eta, \omega\right)\right|^{2}=0$.

Therefore from (2.20) and (2.21) we obtain the inequality

$\mathbf{E}\left|u_{\epsilon}(x, t, \varepsilon, \eta, \omega)-\lambda\right|^{2} \leq \limsup _{N \rightarrow \infty} U_{\epsilon, \boldsymbol{n}}\left(x_{\boldsymbol{n}}, t, \varepsilon, \lambda\right)(2.22)$

In the limit $N \rightarrow \infty$ from Eq.(2.13)-Eq.(2.14) for any fixed $\epsilon \neq 0, \epsilon \ll 1$, we obtain the differential master equationforColombeau-Ito's SPDE (2.3)-(2.4)

$$
\begin{aligned}
& \frac{d\left(U_{\epsilon}(x, t, \varepsilon, \lambda)\right)_{\epsilon}}{d t}+\Delta\left[\varepsilon-(1+\Delta)^{2}\right]\left(U_{\epsilon}(x, t, \varepsilon, \lambda)\right)_{\epsilon}+ \\
& +\lambda \sum_{i=1}^{r} \delta_{i}\left(F_{\epsilon}\left(\frac{\partial U_{\epsilon}(x, t, \varepsilon, \lambda)}{\partial x_{i}}\right)\right)_{\epsilon}+\left(f_{\epsilon}(x, t)\right)_{\epsilon}+O(\epsilon)=0,(2.23) \\
& U_{\epsilon}(x, t, \varepsilon, \lambda)=-\lambda .
\end{aligned}
$$

Therefore from the inequality (2.22) followsthe inequality

$\mathbf{E}\left|u_{\epsilon}(x, t, \varepsilon, \eta, \omega)-\lambda\right|^{2} \leq U_{\epsilon}(x, t, \varepsilon, \lambda)$.

In the limit $\epsilon \rightarrow 0$ from differential equation (2.23)-(2.24)we obtain the differential equation (2.8)-(2.9)and it is easy to see that

$\lim _{\epsilon \rightarrow 0} U_{\epsilon}(x, t, \varepsilon, \lambda)=\Re(x, t, \varepsilon, \lambda)$.

From the inequality (2.25) one obtainthe inequality

$\liminf _{\epsilon \rightarrow 0} \mathbf{E}\left|u_{\epsilon}(x, t, \varepsilon, \eta, \omega)-\lambda\right|^{2} \leq \lim _{\epsilon \rightarrow 0} U_{\epsilon}(x, t, \varepsilon, \lambda)=\Re(x, t, \varepsilon, \lambda) \cdot(2.27)$

From the inequality (2.27) and Eq.(2.26) finally we obtainthe inequality

$\liminf _{\epsilon \rightarrow 0} \mathbf{E}\left|u_{\epsilon}(x, t, \varepsilon, \eta, \omega)-\lambda\right|^{2} \leq \Re(x, t, \varepsilon, \lambda)$.

The inequality (2.28) finalized the proof.

Definition 2.7.(TheDifferential Master Equation)The linear PDE:

$\frac{\partial \Re(x, t, \varepsilon, \lambda)}{\partial t}+\Delta\left[\varepsilon-(1+\Delta)^{2}\right] \Re(x, t, \varepsilon, \lambda)+\lambda \sum_{i=1}^{r} \delta_{i} \frac{\partial \Re(x, t, \varepsilon, \lambda)}{\partial x_{i}}-f(x, t)=0, \lambda \in \mathbb{R},(2.29)$

$\mathfrak{R}(x, 0, \varepsilon, \lambda)=0 \quad(2.30)$,

We will call asthe differential master equation. 
Definition 2.8.(TheTranscendental Master Equation)Thetranscendental equation

$\Re(x, t, \varepsilon, \lambda(x, t, \varepsilon))=0, \quad(2.31)$

wewill call asthe transcendentalmaster equation.

Remark2.2. We note that concrete structure of the Nikolaevskii chaos is determined by the solutions $\lambda(x, t, \varepsilon)$ variety bytranscendentalmaster equation(2.31). Master equation (2.31)is determines by the only way some many-valued function $\lambda(x, t, \varepsilon)$ which is the main constructive object, determining thecharacteristics of quantum chaos in the corresponding model of Euclidian quantum fieldtheory.

\section{Criterion of the existence quantum chaos in Euclidian quantum N-model.}

Definition3.1.Let $u_{\eta}(x, t, \varepsilon, \omega)$ be the solution of the Eq.(2.1). Assume that for almost all points $(x, t) \in$ $\mathbb{R}^{r} \times \mathbb{R}_{+}$(in the sense of Lebesgue-measureon $\mathbb{R}^{r} \times \mathbb{R}_{+}$), there exist a function $u(x, t)$ such that

$\lim _{\eta \rightarrow 0} \mathbf{E}\left[\left(u_{\eta}(x, t, \varepsilon, \omega)-u(x, t)\right)^{2}\right]=0$.

Then we will say that afunction $u(x, t)$ is a quasi-determined solution (QD-solution of the Eq.(2.).

Definition3.2. Assume that there exist a set $\mathfrak{Y} \subset \mathbb{R}^{r} \times \mathbb{R}_{+}$that is positive

Lebesgue-measure, i.e., $\mu(\mathfrak{H})>0$ and

$\forall(x, t)\left\{(x, t) \in \mathfrak{T} \rightarrow \neg \exists \lim _{\eta \rightarrow 0} \mathbf{E}\left[u_{\eta}^{2}(x, t, \varepsilon, \omega)\right]\right\},(3.2)$

i.e., $(x, t) \in \mathfrak{S}$ imply that the $\operatorname{limit} \lim _{\eta \rightarrow 0} \mathbf{E}\left[u_{\eta}^{2}(x, t, \varepsilon, \omega)\right]$ does not exist.

Then we will say thatEuclidian quantum $\mathrm{N}$-model has thequasi-determined Euclidian quantum chaos (QD-quantum chaos).

Definition3.3.For each point $(x, t) \in \mathbb{R}^{r} \times \mathbb{R}_{+}$we define a set $\{\widetilde{R}(x, t, \varepsilon)\} \subset \mathbb{R}$ by the condition:

$\forall \lambda[\lambda \in\{\widetilde{R}(x, t, \varepsilon)\} \Leftrightarrow \Re(x, t, \varepsilon, \lambda)=0] .(3.3)$

Definition3.4.Assume that Euclidian quantum $\mathrm{N}$-model(2.1) has the Euclidian QD-quantum chaos. For each point $(x, t) \in \mathbb{R}^{r} \times \mathbb{R}_{+}$we define a set-valued function $\widetilde{R}(x, t): \mathbb{R}^{r} \times \mathbb{R}_{+} \rightarrow 2^{\mathbb{R}}$ by the condition:

$\widetilde{\mathfrak{R}}(x, t, \varepsilon)=\{\widetilde{\mathfrak{R}}(x, t, \varepsilon)\}(3.4)$

We will say thattheset -valued function $\widetilde{R}(x, t, \varepsilon)$ is a quasi-determinedchaotic solution(QD-chaotic solution)of the quantum $\mathrm{N}$-model. 


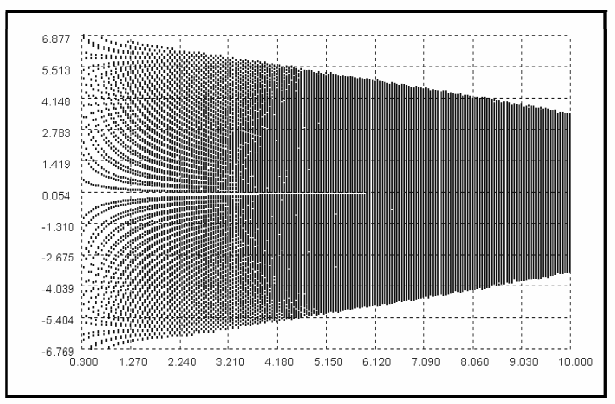

Pic.3.1.Evolution of QD-chaotic solution $\widetilde{R}(x, t, \varepsilon)$ in timet $\in[0,10]$ at point $x=3 . t \in[0,10], \varepsilon=-10^{-2}, \sigma=10^{3}, p=1.1$.

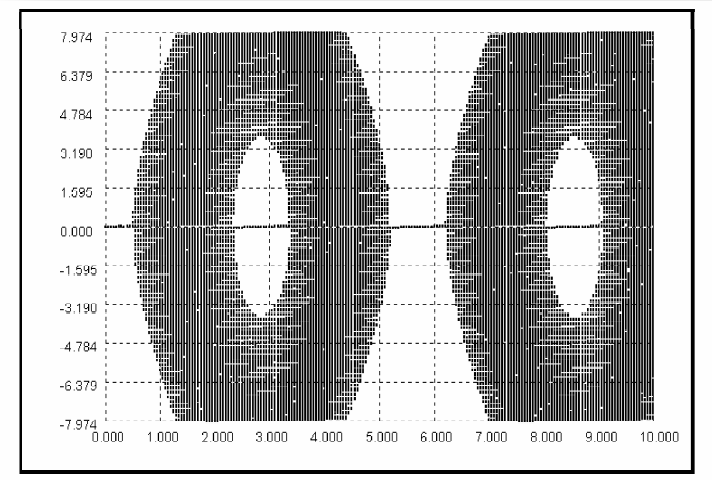

Pic.3.2.The spatial structure ofQD-chaoticsolution $\widetilde{\Re}(x, t, \varepsilon)$ at instant $t=3, \varepsilon=-10^{-2}, \sigma=10^{3}, p=1.1$.

Theorem3.1.Assume that $f(x, t)=\sigma \sin (p \cdot x)$ Then for all values of parameters $r, \varepsilon, \sigma, \delta_{j}, j=1, \ldots, r$ such that $r \in \mathbb{N}, \delta_{j} \in \mathbb{R}_{+}, j=1, \ldots, r, \varepsilon \in[-1,1], p \in \mathbb{R}^{r}, \sigma \neq 0$, quantum $\mathrm{N}$-model (2.1) has the QD-chaotic solutions.

Definition3.5.For each point $(x, t) \in \mathbb{R}^{r} \times \mathbb{R}_{+}$we define the functions such that:

(i) $\quad u_{+}(x, t, \varepsilon)=\limsup _{\eta \rightarrow 0} \mathbf{E}\left[u_{\eta}(x, t, \varepsilon, \omega)\right]$,

(ii) $\quad u_{-}(x, t, \varepsilon)=\liminf _{\eta \rightarrow 0} \mathbf{E}\left[u_{\eta}(x, t, \varepsilon, \omega)\right]$,

(iii) $\quad u_{w}(x, t, \varepsilon)=u_{+}(x, t, \varepsilon, \omega)-u_{-}(x, t, \varepsilon, \omega)$.

\section{Definition3.7.}

(i) Function $u_{+}(x, t, \varepsilon)$ is calledupper boundof the QD-quantum chaosat point $(x, t)$. 
(ii) Function $u_{-}(x, t, \varepsilon)$ is called lower bound of the QD-quantum chaosat point $(x, t)$.

(iii) Function $u_{w}(x, t, \varepsilon)$ is called width oftheQD-quantum chaosat point $(x, t)$.

Definition3.8. Assume now that

$\limsup _{t \rightarrow \infty} u_{w}(x, t, \varepsilon)=u_{w}(x, \varepsilon)<\infty .(3.5)$

Then we will say thatEuclidian quantum N-model has QD-quantum chaos of the asymptotically finitewidthat point $x \in \mathbb{R}^{r}$.

Definition3.9. Assume now that

$\limsup _{t \rightarrow \infty} u_{w}(x, t, \varepsilon)=u_{w}(x, \varepsilon)=\infty$

Then we will say that Euclidian quantum N-model has QD-quantum chaos of the asymptotically infinite width at point $x \in \mathbb{R}^{r}$.

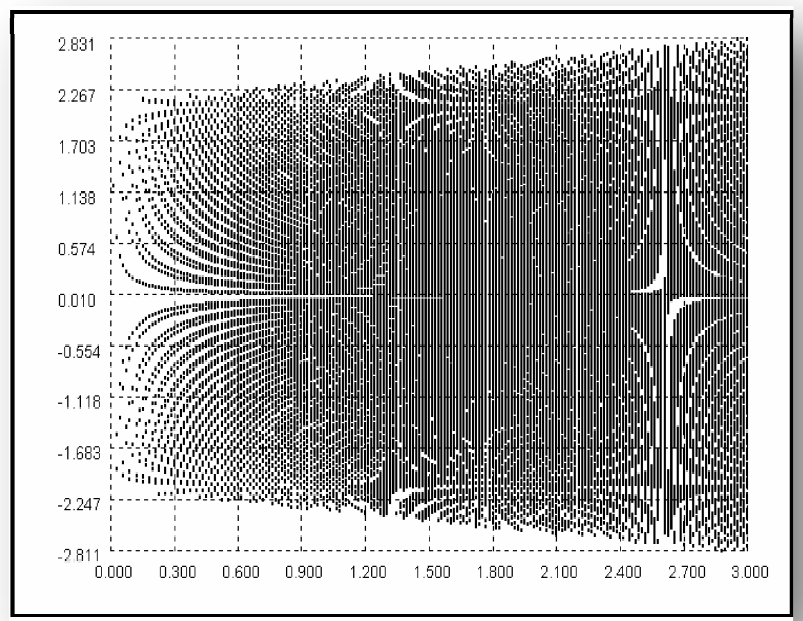

Pic.3.3.TheQD-quantum chaos of the asymptotically infinite width at point $x=3 . \varepsilon=0.1, \delta=10, \sigma=$ $10^{3}, p=1$.

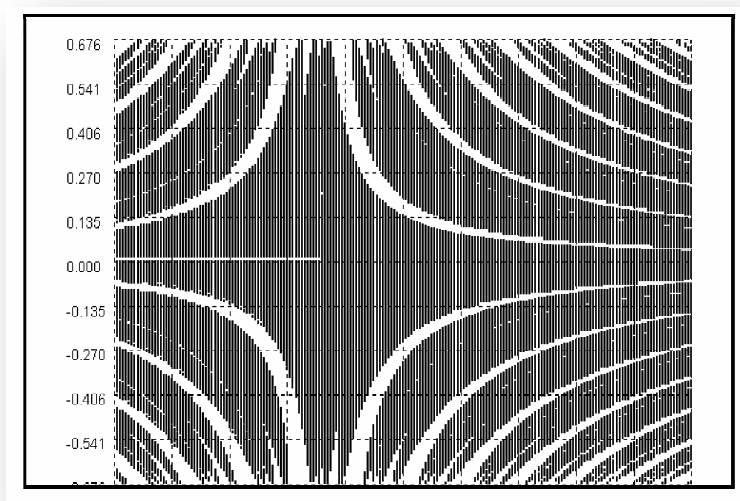


Pic.3.4.The fine structure of the QD-quantum chaos of the asymptotically infinite width at point $x=$

$$
3, \varepsilon=0, \delta=10, \sigma=10^{4}, p=1, t \in\left[10^{4}, 10^{4}+10^{-1}\right], \lambda \in[-0.676,0.676] \text {. }
$$

Definition3.10. For each point $(x, t) \in \mathbb{R}^{r} \times \mathbb{R}_{+}$we define the functions such that:

$$
\begin{array}{ll}
\text { (i) } & \widetilde{\mathfrak{R}}_{+}(x, t, \varepsilon)=\sup \{\widetilde{\mathfrak{R}}(x, t, \varepsilon)\}, \\
\text { (ii) } & \widetilde{\mathfrak{R}}_{-}(x, t, \varepsilon)=\inf \{\widetilde{\mathfrak{R}}(x, t, \varepsilon)\}, \\
\text { (iii) } & \widetilde{\Re}_{w}(x, t, \varepsilon)=\widetilde{\mathfrak{R}}_{+}(x, t, \varepsilon)-\widetilde{\mathfrak{R}}_{-}(x, t, \varepsilon) .
\end{array}
$$

Theorem3.2. For each point $(x, t) \in \mathbb{R}^{r} \times \mathbb{R}_{+}$is satisfiedtheinequality

$\widetilde{R}_{w}(x, t, \varepsilon) \leq u_{w}(x, t, \varepsilon) \cdot(3.7)$

Proof. Immediately follows by Theorem2.1 and Definitions 3.5, 3.10.

\section{Theorem3.3. (Criterion of QD-quantum chaos in Euclidian quantum N-model)}

Assume that

$\operatorname{mes}\left\{(x, t) \mid \widetilde{\Re}_{w}(x, t, \varepsilon)>0\right\}>0 .(3.8)$

Then Euclidian quantum N-model has QD-quantum chaos.

Proof. Immediately follows bytheinequality(3.7)and Definition3.2.

\section{Quasi-determined quantum chaos and physical turbulencenature.}

In generally accepted at the present time hypothesiswhatphysical turbulencein the dynamical systems with an infinite number of degrees of freedom really is, thephysical turbulence is associated with a strangeattractors, on which the phase trajectories of dynamical system reveal the knownproperties of stochasticity: a very high dependence on the initial conditions, whichis associated with exponential dispersion of the initially close trajectories and bringsto their non-reproduction; everywhere the density on the attractor almost of all thetrajectories a very fast decreaseoflocal auto-correlation function[2]-[9]

$\Phi(\mathrm{x}, \tau)=\langle\tilde{u}(x, t) \tilde{u}(x, \tau+t)\rangle,(4.1)$

Here

$$
\tilde{u}(x, t)=u(x, t)-\langle u(x, t)\rangle,\langle f(t)\rangle=\lim _{T \rightarrow \infty}\langle f(t)\rangle_{T},\langle f(t)\rangle_{T}=\frac{1}{T} \int_{0}^{T} f(t) .
$$

In contrast with canonical numerical simulation, by using Theorem2.1 it is possible to study non-perturbativelythe influence of thermal additive fluctuationson classical dynamics, which in the consideredcase is described by equation (4.1).

The physicalnature of quasi-determined chaosis simple andmathematically is associated withdiscontinuously of the trajectories of the stochastic process $u_{\eta}(x, t, \varepsilon, \omega)$ on parameter $\eta$.

In order to obtain thecharacteristics of this turbulence, which is a very similarlytolocal auto-correlation function (3.1) we define bellowsomeappropriatefunctions. 
Definition 4.1.The numbering function $N(t, x)$ of quantum chaos in Euclidian quantum $\mathrm{N}$-modelis defined by

$N(x, t)=\operatorname{card}\{\widetilde{R}(x, t)\} \cdot(4.2)$

Here by $\operatorname{card}\{X\}$ we denote the cardinality of a finite set $X$,i.e., the number of its elements.

Definition 4.2. Assume now that a set $\{\widetilde{R}(x, t)\}$ is ordered be increase of its elements. We introduce the function $\widetilde{\Re}_{i}(x, t), i=1, \ldots, N(x, t)$ which value at point $(x, t)$, equals the $i$-th element ofa set $\{\widetilde{R}(x, t)\}$.

Definition 3.3.The mean value function $\bar{u}(x, t)$ ofthe chaotic solution $\widetilde{R}(x, t)$ at point $(x, t)$ is defined by $\bar{u}(x, t)=(N(x, t))^{-1} \sum_{i=1}^{N(x, t)} \widetilde{R}_{i}(x, t) \cdot(4.3)$

Definition 3.4.The turbulent pulsations function $u^{*}(x, t)$ of the chaotic solution $\widetilde{R}(x, t)$ at point $(x, t)$ is defined by

$u^{*}(x, t)=\sqrt{(N(x, t))^{-1} \sum_{i=1}^{N(x, t)}\left|\widetilde{\Re}_{i}(x, t)-\bar{u}(x, t)\right|}$.(4.4)

Definition3.5.Thelocal auto-correlation function is definedby

$$
\begin{aligned}
& \Phi(x, \tau)=\lim _{T \rightarrow \infty}\langle\tilde{u}(x, \tau) \tilde{u}(x, \tau+t)\rangle_{T}=\lim _{T \rightarrow \infty} \frac{1}{T} \int_{0}^{T} \tilde{u}(x, t) \tilde{u}(x, \tau+t) d t,(4.5) \\
& \tilde{u}(x, t)=\bar{u}(x, t)-\breve{u}(x), \breve{u}(x)=\lim _{T \rightarrow \infty} \frac{1}{T} \int_{0}^{T} \bar{u}(x, t) d t .(4.6)
\end{aligned}
$$

Definition 3.5.Thenormalized local auto-correlation function is defined by

$$
\Phi_{\mathrm{n}}(x, \tau)=\frac{\Phi(x, \tau)}{\Phi(x, 0)}(4.7)
$$

Let us consider now 1DEuclidian quantum $\mathrm{N}$-model corresponding to classical dynamics

$$
\frac{\partial^{2}}{\partial x^{2}}\left[\varepsilon-\left(1+\frac{\partial^{2}}{\partial x^{2}}\right)^{2}\right] u(x, \varepsilon)+\delta \frac{\partial u(x, \varepsilon)}{\partial x} u(x, \varepsilon)-\sigma \sin (p \cdot x)=0,(4.8)
$$

Corresponding Langevin equation are [34]-[35]:

$$
\begin{gathered}
\frac{\partial u_{\eta}(x, t, \varepsilon)}{\partial t}+\Delta\left[\varepsilon-(1+\Delta)^{2}\right] u_{\eta}(x, t, \varepsilon)+\delta \frac{\partial u_{\eta}(x, t, \varepsilon)}{\partial x} u_{\eta}(x, t, \varepsilon)- \\
-\sigma \sin (p x)=\sqrt{\eta} w(x, t), \delta>0 \Delta=\frac{\partial^{2}}{\partial x^{2}},(4.9) \\
u_{\eta}(x, 0, \varepsilon)=0, w(x, t)=\frac{\partial^{2} w(x, t)}{\partial x \partial t} .(4.10)
\end{gathered}
$$

Corresponding differential master equation are 
$\frac{\partial \Re(x, t, \varepsilon, \lambda)}{\partial t}+\Delta\left[\varepsilon-(1+\Delta)^{2}\right] \Re(x, t, \varepsilon, \lambda)+\lambda \delta \frac{\partial \Re(x, t, \varepsilon, \lambda)}{\partial x}-\sigma \sin (p x)=0,(4.11)$

$\Re(x, 0, \varepsilon, \lambda)=-\lambda .(4.12)$

Corresponding transcendental master equation (2.29)-(2.30) are

$\frac{\{\cos (p \cdot x)-\exp [t \cdot \chi(p)] \cos [p(x-\lambda \cdot \delta \cdot t)]\} \cdot \lambda \cdot \delta \cdot p}{\chi^{2}(p)+\lambda^{2} \cdot \delta^{2} \cdot p^{2}}+\frac{\{\sin (p \cdot x)-\exp [t \cdot \chi(p)] \sin [p(x-\lambda \cdot \delta \cdot t)]\} \cdot \chi(p)}{\chi^{2}(p)+\lambda^{2} \cdot \delta^{2} \cdot p^{2}}+\frac{\lambda}{\sigma}=0,(4.13)$

$\chi(p)=p^{2}\left[\varepsilon-\left(p^{2}-1\right)^{2}\right] \cdot(4 \cdot 14)$

We assume now that $\chi(p)=0$.Then from Eq.(4.13) for allt $\in[0, \infty)$ we obtain

$\frac{\{\cos (p \cdot x)-\cos [p(x-\lambda \cdot \delta \cdot t)]\} \cdot \lambda \cdot \delta \cdot p}{\lambda^{2} \cdot \delta^{2} \cdot p^{2}}+\frac{\lambda}{\sigma}=0, \operatorname{or}(4.14)$

$\{\cos (p \cdot x)-\cos [p(x-\lambda \cdot \delta \cdot t)]\} \cdot \sigma \cdot \delta^{-1} \cdot p^{-1}+\lambda^{2}=0 .(4.15)$

The result of calculation using transcendental master equation (4.15) the corresponding function $\widetilde{R}(x, t, \varepsilon)$ is presented by Pic. 4.1 and Pic.4.2.

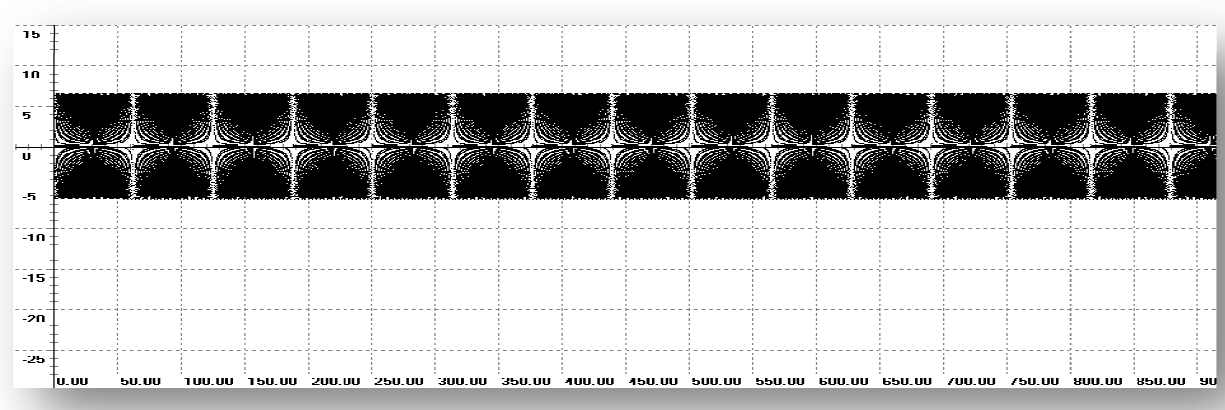

Pic.4.1.Evolution of QD-chaotic solution $\widetilde{R}\left(10^{3}, t, \varepsilon\right)$ in timet $\in\left[0,10^{3}\right], \Delta t=0.1, \varepsilon=0, p=1$,

$$
\sigma=10^{2}, \delta=1, \Delta \lambda=0.01
$$

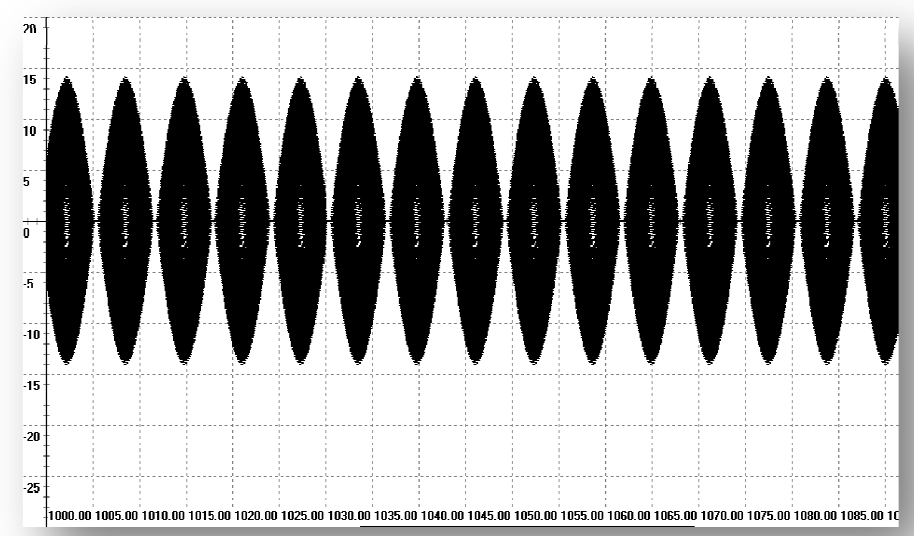


Pic.4.2.The spatial structure ofQD-chaoticsolution $\widetilde{R}(x, t, \varepsilon)$ at instant $t=10^{3}, \varepsilon=0, p=1, \sigma=10^{2}$,

$$
\delta=1, \Delta x=0.1, \Delta \lambda=0.01
$$

The result of calculation using master equation(4.13) the correspondingfunction $\widetilde{R}(x, t, \varepsilon)$ is presented by Pic. 4.3 and Pic.4.4.

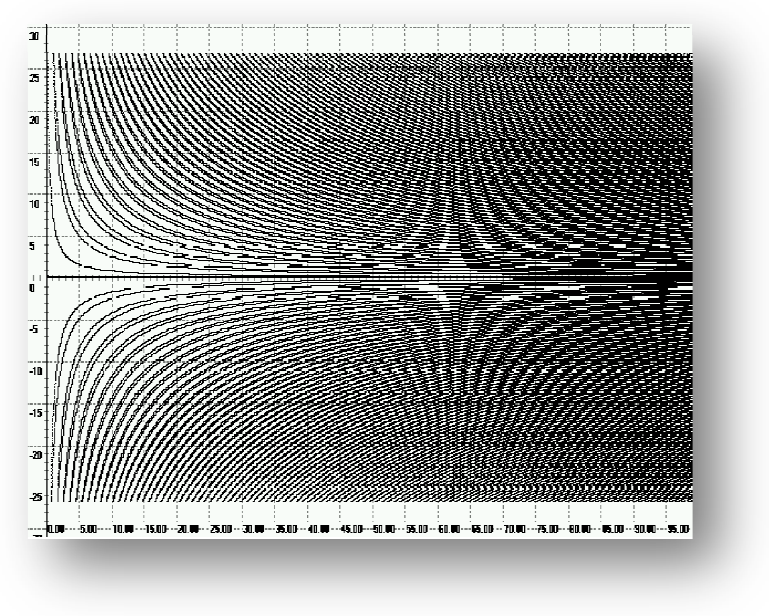

Pic.4.3.Thedevelopment of temporal chaotic regime of 1D EuclidianquantumN-model at point $x=1, t \in\left[0,10^{2}\right] . \varepsilon=10^{-7}, \sigma=10^{2}, \delta=1, p=1$.

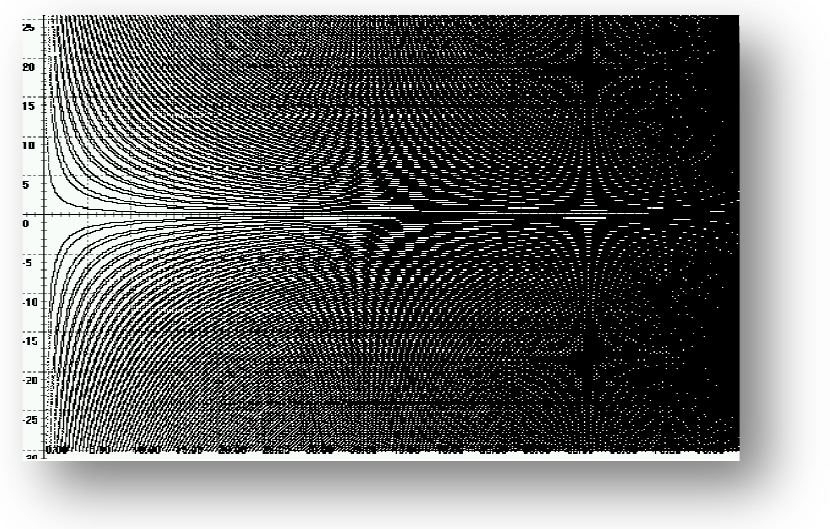

Pic.4.4.The developmentof temporal chaotic regimeof 1D

Euclidian quantum $\mathrm{N}$-model at point $x=1, t \in\left[0,10^{2}\right], \varepsilon=10^{-7}, \sigma=5 \cdot 10^{5}, \delta=1, p=1$.

Let us calculate now correspondingnormalized local auto-correlation function $\Phi_{\mathrm{n}}(x, \tau)$. The result of calculation using Eq.(4.7)-Eq.(4.7) is presented by Pic.4.5 and Pic.4.6. 


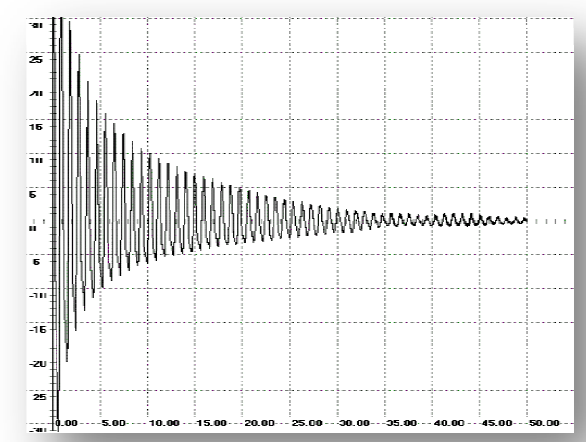

Pic.4.5. Normalized local auto-correlation function $\Phi_{\mathrm{n}}(1, \tau)$ $t \in[0,50], \varepsilon=10^{-7}, \sigma=10^{2}, \delta=1, p=1$.

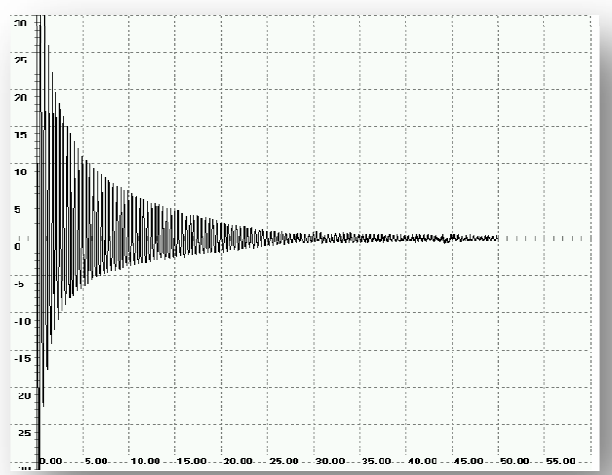

Pic.4.6.Normalized local auto-correlation function $\Phi_{\mathrm{n}}(1, \tau)$. $t \in[0,100], \varepsilon=10^{-7}, \sigma=5 \cdot 10^{5}, \delta=1, p=1$.

Inpaper [7]the mechanism of the onset of chaos and its relationship to the characteristics of the spiral attractors are demonstrated for inhomogeneous media that can be modeled by the Ginzburg- Landau equation(4.14). Numerical data are compared with experimental results.

$$
\begin{aligned}
\frac{\partial a(x, t)}{\partial t}=i \omega(x) a(x, t)+\frac{1}{2}\left(1-|a(x, t)|^{2}\right) a(x, t)+g \frac{\partial^{2} a(x, t)}{\partial x},(4.14) & \\
& \frac{\partial a(0, t)}{\partial x}=0, \frac{\partial a(l, t)}{\partial x}=0, x \in[0, l], l=50
\end{aligned}
$$




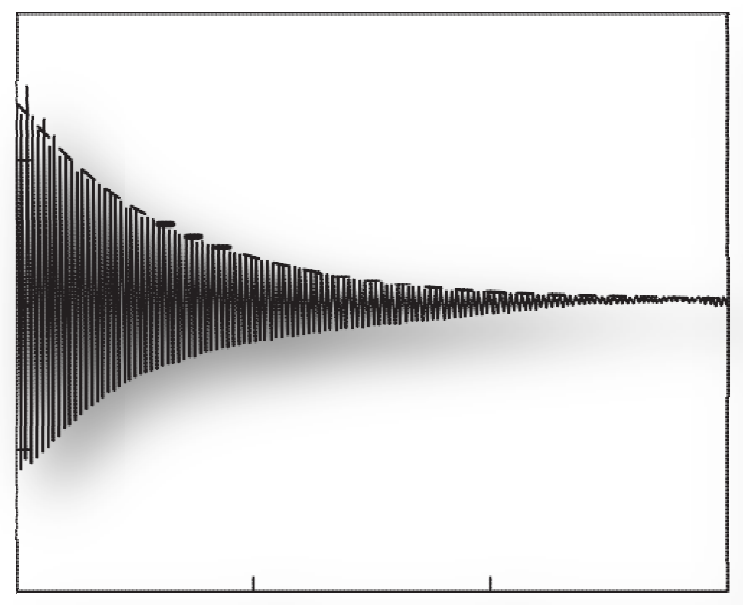

Pic.4.7. Normalized local auto-correlation function $\Phi_{\mathrm{n}}(25, \tau)[7]$.

However as pointed out above (see Remark1.1-1.4 ) such numerical simulation in factgivesnumerical data for stochastic model

$$
\begin{gathered}
\frac{\partial a(x, t)}{\partial t}=i \omega(x) a(x, t)+\frac{1}{2}\left(1-|a(x, t)|^{2}\right) a(x, t)+g \frac{\partial^{2} a(x, t)}{\partial x}+\sqrt{\varepsilon} w(x, t), \varepsilon \ll 1,(4.15) \\
\frac{\partial a(0, t)}{\partial x}=0, \frac{\partial a(l, t)}{\partial x}=0, x \in[0, l], l=50
\end{gathered}
$$

\section{The order of the phase transitionfrom a spatially uniformstate to a turbulent state at instant $t \approx 0$.}

In order to obtain the character of the phase transition (first-order or second-order on parameters $\varepsilon, p$ ) from a spatially uniform to a turbulent stateat instant $t \approx 0$ one can to use the master equation () of the form

$\Re(x, t, \varepsilon, \lambda(x, t, \varepsilon))=0 . \quad(5.1)$

Bydifferentiation the Eq.(5.1) one obtain

$\frac{d \Re(x, t, \varepsilon, \lambda(x, t, \varepsilon))}{d \varepsilon}=\frac{\partial \Re(x, t, \varepsilon, \lambda(x, t, \varepsilon))}{\partial \lambda} \frac{d \lambda(x, t, \varepsilon)}{d \varepsilon}+\frac{\partial \Re(x, t, \varepsilon, \lambda(x, t, \varepsilon))}{\partial \varepsilon}=0 . \quad$ (5.2)

FromEq.(5.2) one obtain

$\frac{d \lambda(x, t, \varepsilon)}{d \varepsilon}=-\left(\frac{\partial \Re(x, t, \varepsilon, \lambda(x, t, \varepsilon))}{\partial \varepsilon}\right) \cdot\left(\frac{\partial \Re(x, t, \varepsilon, \lambda(x, t, \varepsilon))}{\partial \lambda}\right)^{-1}$

Let us consider now 1DEuclidian quantum N-model given byEq. (4.9)-Eq. (4.10). From corresponding transcendental master equation Eq.(4.13)by differentiation the equation Eq.(4.13)with respect to variable $\lambda$ one obtain 


$$
\begin{aligned}
& \frac{\partial \Re(x, t, \varepsilon, \lambda(x, t, \varepsilon))}{\partial \lambda} \\
& =\frac{\{\cos (p \cdot x)-\exp [t \cdot \chi(p)] \cos [p(x-\lambda \cdot \delta \cdot t)]\} \cdot \delta \cdot p}{\chi^{2}(p)+\lambda^{2} \cdot \delta^{2} \cdot p^{2}} \\
& +\frac{\{\cos (p \cdot x)-\exp [t \cdot \chi(p)] \sin [p(x-\lambda \cdot \delta \cdot t)]\} \cdot \lambda \cdot t \cdot \delta^{2} \cdot p^{2}}{\chi^{2}(p)+\lambda^{2} \cdot \delta^{2} \cdot p^{2}}- \\
& -\frac{\{\cos (p \cdot x)-\exp [t \cdot \chi(\mathrm{p})] \cos [p(x-\lambda \cdot \delta \cdot t)]\} \cdot 2 \cdot \lambda \cdot \delta^{3} \cdot p^{3}}{\left[\chi^{2}(p)+\lambda^{2} \cdot \delta^{2} \cdot p^{2}\right]^{2}} \\
& -\frac{\{\sin (p \cdot x)-\exp [t \cdot \chi(p)] \cos [p(x-\lambda \cdot \delta \cdot t)]\} \cdot \delta \cdot t \cdot \chi(p)}{\chi^{2}(p)+\lambda^{2} \cdot \delta^{2} \cdot p^{2}}- \\
& -\frac{\{\sin (p \cdot x)-\exp [t \cdot \chi(p)] \sin [p(x-\lambda \cdot \delta \cdot t)]\} 2 \cdot \lambda \cdot \chi(p) \cdot \delta^{2} \cdot p^{2}}{\left[\chi^{2}(p)+\lambda^{2} \cdot \delta^{2} \cdot p^{2}\right]^{2}}+\frac{1}{\sigma}
\end{aligned}
$$

From Eq.(5.4)for a sufficiently small $t \approx 0$ one obtain

$$
\left[\frac{\partial \Re(x, t, \varepsilon, \lambda(x, t, \varepsilon))}{\partial \lambda}\right]_{t \approx 0}=\frac{1}{\sigma} \cdot(5.5)
$$

From master equation Eq.(4.13) one obtainby differentiation the equation Eq.(4.13)with respect to variable $\varepsilon$ one obtain

$$
\begin{gathered}
\frac{\partial \mathfrak{R}(x, t, \varepsilon, \lambda)}{\partial \varepsilon}=\frac{\left\{-t \cdot \frac{d \chi(p)}{d \varepsilon} \exp [t \cdot \chi(p)] \cos [p(x-\lambda \cdot \delta \cdot t)]\right\} \cdot \lambda \cdot \delta \cdot p}{\chi^{2}(p)+\lambda^{2} \cdot \delta^{2} \cdot p^{2}} \\
-\frac{\{\cos (p \cdot x)-\exp [t \cdot \chi(p)] \cos [p(x-\lambda \cdot \delta \cdot t)]\} \cdot 2 \cdot \frac{d \chi(p)}{d \varepsilon} \cdot \lambda \cdot \delta \cdot p}{\left[\chi^{2}(p)+\lambda^{2} \cdot \delta^{2} \cdot p^{2}\right]^{2}}+ \\
+\frac{\left\{-t \cdot \frac{d \chi(p)}{d \varepsilon} \exp [t \cdot \chi(p)] \sin [p(x-\lambda \cdot \delta \cdot t)]\right\} \cdot \chi(p)}{\chi^{2}(p)+\lambda^{2} \cdot \delta^{2} \cdot p^{2}} \\
+\frac{\{\sin (p \cdot x)-\exp [t \cdot \chi(p)] \sin [p(x-\lambda \cdot \delta \cdot t)]\} \cdot \frac{d \chi(p)}{d \varepsilon}}{\chi^{2}(p)+\lambda^{2} \cdot \delta^{2} \cdot p^{2}} \\
-\frac{\{\sin (p \cdot x)-\exp [t \cdot \chi(p)] \sin [p(x-\lambda \cdot \delta \cdot t)]\} 2 \cdot \chi^{2}(p) \cdot \frac{d \chi(p)}{d \varepsilon}(5.6)}{\left[\chi^{2}(p)+\lambda^{2} \cdot \delta^{2} \cdot p^{2}\right]^{2}}
\end{gathered}
$$

From Eq.(5.6)for a sufficiently small $t \approx 0$ one obtain

$$
\left[\frac{\partial \Re(x, t, \varepsilon, \lambda(x, t, \varepsilon))}{\partial \varepsilon}\right]_{t \approx 0}=-\left(t \cdot \frac{d \chi(p)}{d \varepsilon}\right) \frac{\chi(p) \cdot p \cdot \sin (p \cdot x)}{\chi^{2}(p)+\lambda^{2} \cdot \delta^{2} \cdot p^{2}}=t p^{2}\left(p^{2}-1\right)^{2} \frac{\chi(p) \sin (p \cdot x)}{\chi^{2}(p)+\lambda^{2} \cdot \delta^{2} \cdot p^{2}},(5.7)
$$

Therefore from Eq.(5.3),Eq. (5.5) and Eq.(5.7) one obtain 
$[\Im(\varepsilon, x, t)]_{t \approx 0}=\left[\frac{1}{t} \frac{d \lambda(x, t, \varepsilon)}{d \varepsilon}\right]_{t \approx 0} \approx \sigma p^{2}\left(p^{2}-1\right)^{2} \frac{\sin (p \cdot x)}{\chi(p)} \operatorname{sign}(p \cdot x)$

In the limit $t \rightarrow 0$ from Eq. (5.8) one obtain

$\square(\varepsilon, x)=\lim _{t \rightarrow 0} \frac{d \lambda(x, t, \varepsilon)}{t d \varepsilon}=\sigma p^{2}\left(p^{2}-1\right)^{2} \frac{\sin (p \cdot x)}{\chi(p)} \operatorname{sign}(p \cdot x),(5.9)$

and where $\chi(p)=p^{2}\left[\varepsilon-\left(p^{2}-1\right)^{2}\right]=p^{2} \rho(\varepsilon), \rho(\varepsilon)=\varepsilon-\left(p^{2}-1\right)^{2}$.

FromEq. (5.9) follows that

$\lim _{\rho(\varepsilon) \rightarrow 0_{+}} \square(\varepsilon, x)=+\infty,(5.10)$

$\lim _{\rho(\varepsilon) \rightarrow 0_{-}} \square(\varepsilon, x)=-\infty .(5.11)$

FromEq. (5.10)-(5.11) follows second orderdiscontinuity of the quantity $\square(\varepsilon, x, t)$ at instant $t=0$. Therefore the system causing it to make a direct transitionfrom a spatially uniformstate $u_{\eta \approx 0}(x, 0, \varepsilon)=0$ to a turbulent statein an analogous fashion to the second-order phase transition inquasi-equilibrium systems.

\section{Chaotic regime generatedby periodical multi-modes external perturbation.}

Assume nowthat external periodical force $f(x)$ has the followingmulti-modes form

$f(x)=-\sum_{k=1}^{m} \sigma_{k} \sin \left(p_{k} x\right) \cdot(6.1)$

Corresponding transcendental master equation are

$$
\begin{array}{r}
\square(x, t, \varepsilon, \lambda)=\sum_{k=1}^{m} \sigma_{k} \frac{\left\{\cos \left(p_{k} \cdot x\right)-\exp [t \cdot \chi(p)] \cos \left[p_{k}(x-\lambda \cdot \delta \cdot t)\right]\right\} \cdot \lambda \cdot \delta \cdot p_{k}}{\chi^{2}\left(p_{k}\right)+\lambda^{2} \cdot \delta^{2} \cdot p_{k}^{2}}+ \\
+\sum_{k=1}^{m} \sigma_{k} \frac{\left\{\sin \left(p_{k} \cdot x\right)-\exp \left[t \cdot \chi\left(p_{k}\right)\right] \sin \left[p_{k}(x-\lambda \cdot \delta \cdot t)\right]\right\} \cdot \chi\left(p_{k}\right)}{\chi^{2}\left(p_{k}\right)+\lambda^{2} \cdot \delta^{2} \cdot p_{k}^{2}}+\lambda=0, \chi(p)=p^{2}\left[\varepsilon-\left(p^{2}-1\right)^{2}\right] .(6.2)
\end{array}
$$

Let us consider the examples of QD-chaotic solutions with a periodical force:

$f(x)=-\sigma \sum_{k=1}^{m} \sin \left(\frac{k x}{n}\right) \cdot(6.3)$ 


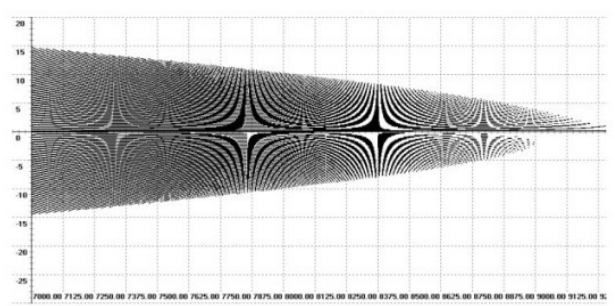

Pic.6.1.Evolution of QD-chaotic solution $\widetilde{\square}\left(10^{3}, t, \varepsilon\right)$ in timet $\in\left[7 \cdot 10^{3}, 10^{4}\right]$, $\Delta t=0.1, m=1, n=100, \varepsilon=-1, p=1, \sigma=10^{2}, \delta=1, \Delta \lambda=0.01$.

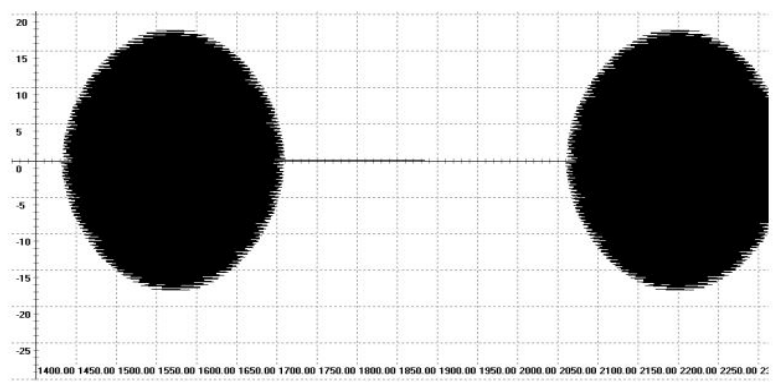

Pic.6.2.The spatial structure ofQD-chaoticsolution $\widetilde{\square}(x, t, \varepsilon)$ at instant $t=10^{3}$, $x \in\left[1.4 \cdot 10^{3}, 2.5 \cdot 10^{3}\right], m=1, n=100, \varepsilon=-1, p=1, \sigma=10^{2}, \delta=1, \Delta x=0.1, \Delta \lambda=0.01$.

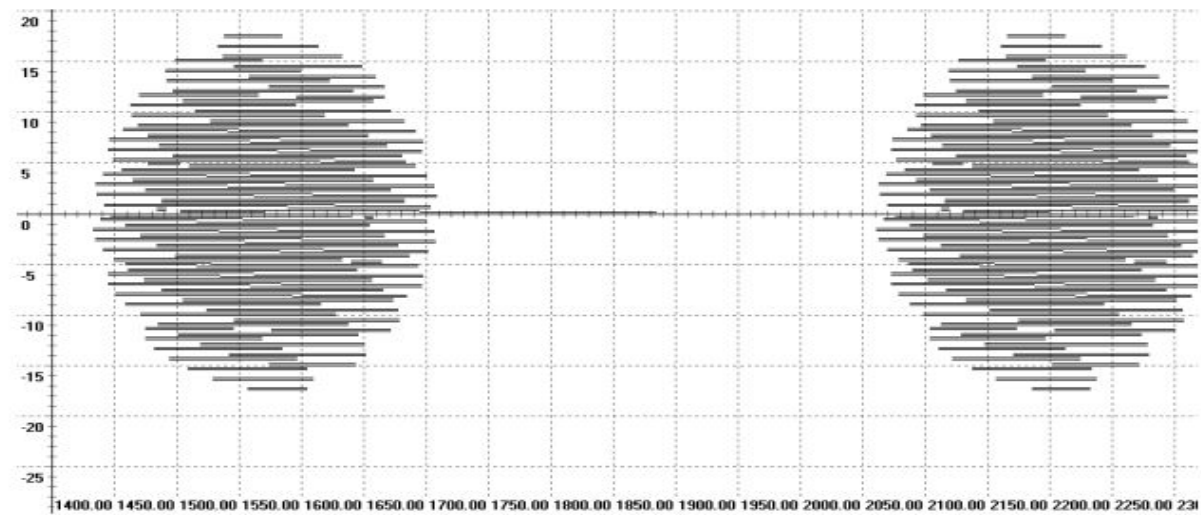

Pic.6.2.The spatial structure ofQD-chaoticsolution $\widetilde{\square}(x, t, \varepsilon)$ at instant $t=5 \cdot 10^{3}$, $x \in\left[1.4 \cdot 10^{3}, 2.5 \cdot 10^{3}\right], m=1, n=100, \varepsilon=-1, p=1, \sigma=10^{2}, \delta=1$, $\Delta x=0.1, \Delta \lambda=0.01$.

\section{Conclusion}

A non-perturbative analytical approach to the studying of problemof quantum chaos in dynamical systems withinfinite number of degrees of freedom isproposed and developed successfully.It is shown that the additive thermal noise destabilizes dramatically the ground state of the system thus causing it to make a direct transition from a spatially uniform to a turbulent state. 


\section{Acknowledgments}

A reviewer provided important clarifications.

\section{References}

[1] Nikolaevskii, V. N.(1989). Recent Advances inEngineering Science, edited by S.L. Kohand C.G. Speciale.Lecture Notes in Engineering, No. 39(Springer - Verlag. Berlin. 1989), pp. 210.

[2] Tribelsky, M. I., Tsuboi, K. (1996).Newscenario to transition to slow turbulence. Phys.Rev. Lett. 761631 (1996).

[3] Tribelsky, M. I. (1997). Short-wavelegthinstability and transition in distributed systems with additional symmetryUspekhifizicheskikhnauk (Progresses of the Physical Studies)V167,N2.

[4] Toral, R., Xiong, J. D. Gunton, and H.W.Xi.(2003) Wavelet Description of the Nikolaevskii Model.Journ.Phys.A 36, 1323 ( 2003).

[5] Haowen Xi, Toral, R,.Gunton, D, Tribelsky M.I. (2003).ExtensiveChaos in the NikolaevskiiModel.Phys. Rev. E.Volume: 61,Page:R17,(2000)

[6] Tanaka, D., Amplitude equations of Nikolaevskii turbulence, RIMS KokyurokuBessatsu B3(2007), 121-129

[7] Fujisaka,H.,Amplitude Equation ofHigher-Dimensional Nikolaevskii Turbulence Progress of Theoretical Physics, Vol. 109, No. 6, June 2003

[8] Tanaka, D.,Bifurcation scenario to Nikolaevskii turbulence in small systems, http://arxiv.org/abs/nlin/0504031v1DOI: 10.1143/JPSJ.74.222

[9] Anishchenko, V. S.,Vadivasova, T. E.,Okrokvertskhov, G. A. and Strelkova,G. I., Statistical properties of dynamical chaos, Physics-Uspekhi(2005),48(2):151 http://dx.doi.org/10.1070/PU2005v048n02ABEH002070

[10] Tsinober, A., The Essence of Turbulence as a Physical Phenomenon,2014, XI, 169 pp., ISBN 978-94-007-7180-2

[11] Ivancevic,V. G.,High-Dimensional Chaotic and Attractor Systems: A Comprehensive Introduction 2007, XV, $697 \mathrm{p}$.

[12] Herbst,B. M. and Ablowitz, M. J.,Numerically induced chaos in the nonlinear Schrödinger equation,Phys. Rev. Lett. 62, 2065

[13] Mark J. Ablowitz and B. M. Herbst,On Homoclinic Structure and Numerically Induced Chaos for the Nonlinear Schrodinger Equation,SIAM Journal on Applied Mathematics Vol. 50, No. 2 (Apr., 1990), pp. 339-351

[14] Li, Y., Wiggins, S., Homoclinic orbits and chaos in discretized perturbed NLS systems: Part II. Symbolic dynamics,Journal of Nonlinear Science1997, Volume 7, Issue 4, pp 315-370.

[15] Blank,M. L., Discreteness and Continuity in Problems of Chaotic Dynamics, Translations of Mathematical Monographs1997; 161 pp; hardcoverVolume: 161ISBN-10: 0-8218-0370-0

[16] FADNAVIS,S., SOME NUMERICAL EXPERIMENTS ON ROUND-OFF ERROR GROWTH IN FINITE COMPUTATION.HTTP://ARXIV.ORG/ABS/PHYSICS/9807003V1

[17] Handbook of Dynamical Systems, Volume 2Handbook of Dynamical Systems Volume 2, Pages 1086 (2002)Edited by Bernold Fiedler ISBN: 978-0-444-50168-4

[18] Gold, Bernard, and Charles M. Rader. "Effects of quantization noise in digital filters." Proceedings of the April 26-28, 1966, Spring joint computer conference. ACM, 1966.

[19] Bennett, W. R., "Spectra of Quantized Signals,"Bell System Technical Journal, vol. 27, pp. 446-472 (July 1948)

[20] I. G. Vladimirov, I. G.,Diamond,P.,A Uniform White-Noise Model for Fixed-Point Roundoff Errors in Digital Systems, Automation and Remote ControlMay 2002, Volume 63, Issue 5, pp 753-765

[21] Möller,M. Lange,W.,Mitschke, F., Abraham, N.B.,Hübner, U.,Errors from digitizing and noise in estimating attractor dimensionsPhysics Letters AVolume 138, Issues 4-5, 26 June 1989, pp. 176-182

[22] Widrow, B. and Kollar, I., Quantization Noise: Round off Error in Digital Computation, Signal 
Processing,Control, and Communications,ISBN: 9780521886710

[23] Foukzon, J.,Advanced numerical-analytical methods for path integral calculation and its application to somefamous problems of 3-D turbulence theory. New scenario for transition to slow turbulence. Preliminary report.Meeting:1011, Lincoln, Nebraska, AMS CP1, Session for Contributed Papers. http://www.ams.org/meetings/sectional/1011-76-5.pdf

[24] Foukzon, J.,New Scenario for Transition to slow Turbulence. Turbulencelike quantum chaosin three dimensional model of Euclidian quantumfield theory.Preliminary report.Meeting:1000, Albuquerque, New Mexico, SS 9A, Special Session on Mathematical Methods in Turbulence. http://www.ams.org/meetings/sectional/1000-76-7.pdf

[25] Foukzon, J.,New scenario for transition to slow turbulence. Turbulence like quantum chaos in three dimensional modelof Euclidian quantum field theory.http://arxiv.org/abs/0802.3493

[26] Foukzon, J.,Communications in Applied Sciences, Volume 2, Number 2, 2014, pp.230-363

[27] Foukzon, J., Large deviations principles of Non-Freidlin-Wentzell type,22 Oct. 2014. http://arxiv.org/abs/0803.2072

[28] Colombeau, J., Elementary introduction to new generalized functions Math. Studies 113, North Holland, 1985

[29] CATUOGNO,P., AND OLIVERA C.,STRONG SOLUTION OF THE STOCHASTIC BURGERS EQUATION, HTTP://ARXIV.ORG/ABS/1211.6622V2

[30] Oberguggenberger, M.,.F.Russo, F.,Nonlinear SPDEs: Colombeau solutions and pathwise limits, HTTP://CITESEERX.IST.PSU.EDU/VIEWDOC/SUMMARY?DOI=10.1.1.40.8866

[31] WALSH, J. B.,FINITE ELEMENT METHODS FOR PARABOLIC STOCHASTIC PDE'S, POTENTIAL ANALYSIS, VOLUME 23, ISSUE 1, PP 1-43.HTTPS://WWW.MATH.UBC.CA/ WALSH/NUMERIC.PDF

[32] Suli,E.,Lecture Notes on Finite ElementMethods for Partial DifferentialEquations, University of Oxford, 2000.http://people.maths.ox.ac.uk/suli/fem.pdf

[33] Knabner, P.,Angermann, L.,Numerical Methods forElliptic and Parabolic PartialDifferential Equations, ISBN 0-387-95449-Xhttp://link.springer.com/book/10.1007/b97419

[34] DIJKGRAAF, R., D. ORLANDO, D. ORLANDO, REFFERT, S., RELATING FIELD THEORIES VIA STOCHASTIC 10.1016/j.nuclphysb.2009.07.018

[35] MASUJIMA,M.,PATH INTEGRAL QUANTIZATION AND STOCHASTIC QUANTIZATION,EDITION: 2ND ED. 2009ISBN-13: 978-3540878506 